\title{
Nog het begin van het haringkaken
}

\author{
te Biervliet
}

In 1405, met de dood van hertogin Margaretha, die vrouwe van Biervliet was, blijkt er geen einde aan de verplichting de Vlaamse kaakharing in de Biervlietse haven te lossen te zijn gekomen. Zoals gezegd, bedroeg deze aanvoer in 139895 last of 1140 tonnen, in 1399 50 last of 600 tonnen en in 1403 amper 30 last of 360 tonnen (1). In 1402 nochtans hadden ook de stuurlui van Nieuwpoort, Oostende en Sluis toelating bekomen de haring op zee te kaken, op voorwaarde hun product te Biervliet te lossen en er, zoals de vissers van die stad, het "nobelgheld ", welke één nobel per last kaakharing bedroeg, te betalen (2). Deze dubbele verplichting, evenals de afwijzende houding van de viskooplui in deze kwestie, blijkt voor de zeelieden van de kusthavens een grote hinderpaal te zijn geweest (3). Eerder dan te

(1) R. Degryse, Het begin van het haringkaken te Biervliet, in Hand. Soc. d'Emul., dl XCV (1958), blz. 72-81.

(2) Id., Oorsprong van het Haringkaken in Vlaanderen, in $\mathrm{Ne}$ derlandsche Historiebladen, dl. I (1938), blz. 201-219. Zie blz. 208.

(3) In een stuk van 2 december 1418, uitgaande van hertog Jan zonder Vrees en betreffende de heffing van het "nobelgheld" en het recht op het "hoepembier" te Duinkerke en te Grevelinge, lezen we het volgende : , ...de la partie de notre bien ame Berthelemy le Voocht, conseiller de notre dit seigneur et son receveur general... nous a este expose en complayant, disant que comme en temps passe les bonnes gens de la ville de Biervliet, appartenant a mon dit seigneur et pere, eussent en privilege, que nulz pescheurs, feussent du pays de Flandres ou autres estrangiers, de quelque estat ou condicion, quilz eussent este ou feussent, ne pouoient mener leur herenc, quilz avoient fait cacquie et salle, tans sur la mer et le strom de Flandres, comme dehors, appelle contrefait harenc, a nul des pors de Flandre, excepte seulement audit Biervliet, ou estoit lestaple dicelui harenc, et que illec len a recue et recevoit pour lors au prouffit de notre dit seigneur et 
Biervliet nog hun in tonnen geslagen haring aan wal te brengen, deden ze voortaan Vlissingen in Zeeland aan en brachten er hun product aan de man, gebruik, dat wellicht tot de tijd van Willem Beukel opklom.

Naast de moeilijkheden, die de vrije aanvoer van eigen tonharing in Vlaanderen in de weg stonden en het gevolg van het verzet van bepaalde kringen van kooplieden waren, was daar ook nog de werkelijk geringe productie van dit product. Uit een nagenoeg gelijktijdig document vernemen we inderdaad, dat sommige Vlaamse stuurlui op zee tijdens het eigenlijk seizoen, dat jaarlijks van 24 augustus tot 21 september duurde, somtijds slechts enkele tonnen haring, hoogstens elf tot twaalf, per schip kaakten (4). Dit wijst on-

pere, comme de ses predecesseurs, de chacun last ung noble dor de Flandre et de ce lesdits de Biervliet et icellui notredit seigneur et pere eussent joy et possesse paisiblement de tel et de si longtemps, quil nest memoire de contraire. Et est advenu que depuis notre dit seigneur et pere, alumble requeste et supplicacion desdits pescheurs de Flandres, considerant les grans fraiz, perte et dangiez, quilz avoient eu et auroient, parce quil leur avoit convenu et convenoit, depuis le temps que les terres environ Biervliet furent inondees dela mer, mener leur dit harenc audit Biervliet, ou les marchans aloient enuis et agrant peril pour achater ledit harenc, parcequelle est de toutes pars advironnee dela mer ...Ce est par meure deliberacion de conseil ordonne, accorde et donne congie et licence a toutes manières de pescheurs, tant de sondit pays de Flandres, comme autres estrangiers, quilz peussent mener lesdits harenc contrefait a tous les pors de Flandres, assavoir audit Biervliet, a Lescluse, a Neufport, a Dunkerke, Gravelinghes et ailleurs, parmi ce quilz soient tenus de luy payer a son prouffit de chacun lest ung noble dor comme par avant il le prenoit audit lieu de Biervliet...". Rijsel, Archives départementales du nord, nr B 1282 (15.407). Papieren schrijfboek met afschriften van stukken betreffende de heffing van het "nobelgheld" in de periode 1402-1423, laatste stuk. Zie ook volgende voetnoot.

(4) „Et il soit ainsi que souventeffoiz il advient, que lesdits pescheurs ou les aucun deulx, qui en ladite saison ont prins du herenc frez, salent et caquent icellui sur la mer les ungs plus les autres moins et aucunefoiz non font en ung voiage que dix ou douze tonneaulz et arrivent chargiez de leur herenc fres ensemble de cellui que ainsi ilz ont sale et caque, les aucuns a Neufport, les autres a Oostende et les autres au port de Lescluse ou desdits lieux il leur convient envoier ledit herenc caque a grant dangiez et despense en notre dite ville de 
getwijfeld op de voortzetting van de oorspronkelijke sluikse praktijken of , frauden, die de visschers daden in tvanghen van den haringhe" (5). De Vlaamse kaakharing werd inderdaad nog steeds als "contrefait", als een navolging van het Schonens product, aangevoerd door de Duitse Hanze beschouwd. De vissers zelf beschouwden het kaken nog altijd als een bijverdienste. We mogen dan ook veronderstellen, dat oorspronkelijk het initiatief van het kaken van de zeelui zelf en niet van hun gebeurlijke opdrachtgevers, meer bepaald de reders, die inderdaad toen reeds bestonden, moet uitgegaan zijn.

Van 1408 af werd door de stuurlui opnieuw gepoogd te Biervliet kaakharing aan te voeren (6). Enkele stuurlui, die met hun schepen vóór Vlissingen aangekomen waren, vroegen alsdan aan de baljuw van Biervliet in de haven aldaar hun tonharing te mogen lossen om haar aan Brabanders te verkopen, wat ze, mits betaling van het "nobelgheld", verkregen. Van de vier stuurlui, die daarvoor in aanmerking kwamen, waren er twee herkomstig uit Biervliet, één uit Hughevliet en één waarschijnlijk uit Koksijde bij Sluis. Ze voerden respectievelijk 6 tonnen, 12 tonnen, 18 tonnen en 3 last of 36 tonnen kaakharing aan (7). Op te merken is, dat de stuurman uit Hughevliet, die met zijn naam vernoemd

Biervliet et mainteffoiz pour ce dangiez delaissent a caquer de leur dit frez herenc, parquoy le noble que nous avons sur le lest dicellui herenc vendu audit Biervliet, ne nous revient pas a se grant valeurs, quil seroit en esdits lieux de Neufport, de Lescluse et d'Ostende..." Ibidem. Stuk van 7 maart 1412. Ordonnantie van Jan zonder Vrees, waardoor voortaan Vlaamse, Zeeuwse en Hollandse kaakharing in de verschillende havens van het graafschap mocht gelost worden, mits storting van het "nobelgheld". Zie bewijsstuk nr IV.

(5) Brugge, Stadsarchief, Stadsrekening van 1396.

(6) Volgens de Nieuwpoortse stadsrekeningen deden de Nieuwpoortenaars in 1406, 1408, 1409 en 1411 Biervliet aan. Ze weigerden er zekere tollen te betalen, wat aanleiding tot een conflict tussen de stad Nieuwpoort en de hertogelijke tollenaar van Biervliet gaf. Nieuwpoort, Oud archief, nrs 3500, 3502, 3503, 3505, rubrieken van de dagvaarten en van de boden.

(7) Zie bewijsstuk ar 1 , 
wordt, niemand minder was dan Jehan Danckaerd, de vroegere gezel van Willem Beukel, de zeerover en visser uit dezelfde plaats (8). Deze vaststelling is ongetwijfeld van belang voor de identificatie van de ware Beukel als "den eersten, die den tonharynck vynck ende sautte" (9).

Voor het jaar 1409 beschikken we over geen cijfers in zake de aanvoer van kaakharing te Biervliet. In 1410 zien we een paar stuurlui, namelijk één uit Koksijde bij Sluis en één uit Blankenberge, door de waterbaljuw van het Zwin beboet worden, omdat ze, tegen de hertogelijke ordonnantie van 1399 in, te Vlissingen hun kaakharing gelost hadden, zonder daarvoor te Biervliet het verschuldigde "nobelgheld" te hebben betaald. Nogmaals gold het hier kleine hoeveelheden, respectievelijk 9 tonnen en $31 / 2$ last of 42 tonnen (10).

Op 9 september 1409 had de stad Biervliet voor haar vissers opnieuw toelating voor de aanvoer van tonharing verkregen (11). Dit zette de andere Vlaamse kuststeden, namelijk Nieuwpoort en Sluis, er toe aan voor hun stuurlui eveneens de vergunning te verkrijgen de op zee gekaakte haring in eigen haven te mogen lossen. Eerst in maart 1412 kwam hiervan iets terecht. Hertog Jan zonder Vrees vergunde alsdan de aanvoer niet alleen van Vlaamse, maar ook van Zeeuwse en Hollandse

(8) Willem Beukel van Hughevliet en zijn gezellen, waaronder Jehan Danckaerd, worden in twee stukken, respectievelijk van 7 januari 1390 en 17 april 1391, in verband met de gevangenneming op zee in 1388 van de Portugese infant Don Diniz, vermeld. Jehan Danckaert is dan nog "le jeune " of "Janssone ". Rijsel, Archives départementales du Nord, nrs B 856/15670 en B 1849/50.507. De heer P. de Saint-Aubin, archivaris te Rijsel, was zo goed voor mij het eerste stuk, dat onleesbaar geworden is, gedeeltelijk te ontcijferen. In dit document, welke evenals het tweede, in het Frans gesteld is, komt de naam "Willem Buckel " voor.

(9) Marcus van Vaernewijck, Die waerachtighe Geschiedenisse van ...Carolus de Vijfde, Gent, 1564.

(10) Zie bewijsstuk nr II.

(11) „en notre ville de Biervliet, qui de ce ont obtenu grace et ottroy de nous par nos lettres patentes donnees en notre ville de Bruges le 7 me jour de septembre lan $1409 \ldots$.. Stuk van 7 maart 1412. Zie bewijsstuk $\mathrm{nr}$ IV. 
tonharing, zowel te Biervliet, als in de kusthavens Sluis, Oostende en Nieuwpoort. Voorwaarde daartoe bleef evenwel steeds de storting van één nobel per last aangevoerde kaakharing, evenals het verbod van invoer van het product in het graafschap. De stuurlui mochten nochtans, zoals dit reeds door de vroegere hertogelijke ordonnantie van 1399 bepaald geworden was, bij storm of ander onheil, hun op zee gekaakte haring in Engeland lossen en aan de man brengen, mits daarvoor bij hun terugkeer in Vlaanderen het verschuldigde , nobelgheld" te betalen (12).

Over de aanvoer van Vlaamse tonharing te Biervliet in 1412 en eerstvolgende jaren zijn we, dank zij enkele rekeningen, ingelicht. In de rekening van Jehan Rugheroc, die in eerstgenoemd jaar als tollenaar en heffer van het "nobelgheld" in deze stad optrad, vinden we een achttal posten met de opsomming van de ladingen geloste tonharing. De rekening vernoemt vier stuurlui uit Biervliet, één uit Hughevliet, één uit Koksijde bij Sluis, één uit Heist en één uit Blankenberge, die tweemaal aangehaald wordt. De totale aanvoer bedroeg 15 last en 11 tonnen of 191 tonnen. De hoogste lading was die van de reeds genoemde Jehan Danckaerd uit Hughevliet, bestaande uit 4 last en 3 tonnen of 51 tonnen (13).

In 1413 verkregen de schepenen van Biervliet voor hun stad een nieuw, mandement vanden stapel vanden tonharing" (14). Dit belette de vissers evenwel niet steeds verder te Vlissingen hun kaakharing te lossen en

(12) Bewijsstuk nr IV.

(13) Bewijsstuk nr III.

(14) "Item vanden mandemente gheimpetreert an onsen gheduchten here ende prinche omme vernieuwet te hebbene, te wetene tmandement vanden stapel van den tonharing, hem ghegheven over den zeghel 4 guldine cronen, loopen te 43 groten tstic, 14 s. 4 d. groten ". Brussel, Alg. rijksarchief, Rekenkamer, nr 32068. Stadsrekening van Biervliet over het jaar 1 mei 141330 april 1414, rubriek "Uutgheven van diversen dinghen ". Zie ook verder passim in dezelfde rekening. 
te verkopen (15). Bovendien ijverden de andere Vlaamse kuststeden voor de volledige afschaffing van het verbod op de invoer van de geloste kaakharing in Vlaanderen zelf (16). In de rekeningen van de baljuw van Nieuwpoort van 1413 en 1414 vinden we de cijfers van de aanvoer van tonharing door de stuurlui van die stad, evenals van Lombardsijde, Raversijde en Oostende in de IJzerhaven. Deze aanvoer was de volgende (17):

Jaar Aantal stuurlui Hoeveelheid geloste kaakharing

$\begin{array}{llll}1413 & 13 & 28 \text { last } & \text { of } 336 \text { tonnen } \\ 1414 & 20 & 31 \text { last en } 6 \text { tonnen of } 372 \text { tonnen }\end{array}$

Van 1413 tot 1416 was het de baljuw, die te Biervliet het "nobelgheld" inde. In zijn rekeningen vinden we naast de namen van de stuurlui nog steeds de cijfers van de geloste hoeveelheden en van het gestorte "nobelgheld" aangeduid, evenwel zonder nadere vermelding van de plaats van herkomst, Biervliet of Hughevliet, van de betrokken zeelui. Deze cijfers zijn (18):

(15) "Item, Jan Boutshooft, die ghesonden was ter Sluus omme te sprekene metten cooplieden vanden haringhe omme een dachvaert te houdene teghadere omme de visschers, die te Vlissinghe hare harinc vercopen, inwelke voyaige hij lette 3 daghen te $20 \mathrm{~s}$. groten sdaechs te wedde, loopt 6 s. groten ". Ibidem, nr 32069. Biervlietse stadsrekening van 1 mei 1415 tot 30 april 1416, onder de rubriek van de "voyaigen". Aldaar is er ook sprake van "tachtervoch vanden tonharinghe".

(16) Nieuwpoort. Oud archief, nrs $3502,3503,3506$, 3507, stadsrekeningen van de jaren 1408, 1409, 1412 en 1413. Rubrieken van de dagvaarten en de boden.

(17) Brussel. Alg. rijksarchief, Rekenkamer, nr 14283 : rekeningen van de baljuw van Nieuwpoort, Zeger van Belle (Soehier de Baelleul), over de periodes 18 sept. 1413-8 jan. 1414 en 17 sept. 1414-14 jan. 1415, onder de rubriek: "Resepte de herenc caque contrefait fait en la mer entre la Saint Bertelmiu et la Saint Mahieu et vendu en la dite ville pour mener hors du pais de Flandres, parmy paijant de chacun lest (1 noble) (vervangen door :) $3 \mathrm{lb}$. forte monnaie, 12 tonneaux pour le lest". Het aantal stuurlui, die vernoemd worden, zijn in de eerste rekening 3 uit Nieuwpoort, 1 uit Lombardsijde, 7 uit Raversijde en 2 onbekende en in de tweede rekening 5 uit Nieuwpoort, 3 uit Lombardsijde, 9 uit Raversijde en 3 uit Oostende.

(18) Brussel, Alg. rijksarchief, Rekenkamer, $\mathrm{nr} 13662$ : rekeningen van de baljuw Clais Utenhove, tevens kapitein van Biervliet, 
Jaar Aantal stuurlui Hoeveelheid geloste kaakharing

$\begin{array}{lll}1413 & 11 & 24 \text { last en } 2 \text { tonnen of } 290 \text { tonnen } \\ 1414 & 12 & 38 \text { last en } 6 \text { tonnen of } 462 \text { tonnen } \\ 1415 & 11 & 41 \text { last } 492 \text { tonnen } \\ 1416 & 12 & 45 \text { last en } 6 \text { tonnen of } 546 \text { tonnen }\end{array}$

Deze verschillende cijfers - die voor Sluis ontbreken - tonen aan, dat de aanvoer van Vlaamse tonharing, alhoewel in stijgende lijn gaande, toch zeer beperkt bleef. Slechts enkele stuurlui waagden zich aan het kaken op zee. Het initiatief onder "fraude " enkele tonnen haring te kaken en aan te voeren kan dus aanvankelijk wel van een eenvoudig stuurman of visser als Willem Beukel van Hughevliet (19) of Jacob Kien van Oostende (20) uitgegaan zijn. Gezien we Jehan Danckaert, de vroegere gezel van Willem Beukel voornoemd, in 1408, 1412 en 1413 (21) te Biervliet kaakharing zien aanvoeren, mogen we wellicht hieruit besluiten, dat hij niets anders deed dan voortzetten, wat hij van zijn oudere makker reeds vóór 1400 geleerd had. Willem Beukel, de visser en zeerover uit Hughevliet, kan dus met het begin van het haringkaken in Vlaanderen wel iets te maken gehad hebben.

\section{R. DEGRYSE}

over de jaren 1413 tot en met 1416, onder de rubriek „Recepte de tout le herenc quaque, fait et cacque par les pescheurs de la ville de Biervliet en amenet oudit lieu entre le jour St Beltelmieu et le jour Saint Mathieu lan mil 400 et..., dont monsieur prent ung noble de chacun last, le noble compte a $5 \mathrm{~s}$. de gros monnaie de Flandres ".

(19) R. Degryse, Willem Beukel(s) van Hughevliet, in de Vlaamse Gids, 38ste jaar, nr 7 (juli 1954), blz. 403-410.

(20) Van Jacob Kien, als zeeman en zeerover, is er sprake in de Nieuwpoortse stadsrekening van 1404: „den 23e dach in meye te Brucghen an de wet omme dat Jacob Kien Inghelschen ghevanghen adde ende tgoed ter Niepoort anghebrocht adde, elc 3 daghen, elc $28 \mathrm{~s}$. daghes, 8 lb. 8 s." .Nieuwpoort, Oud archief, $\mathrm{nr}$ 3498. - Ook in de Oostendse stadsrekeningen komt de naam van Jacob Kien voor.

(21) "De Jehan Dankart de 9 barilx de herenc, 3 s. 9 d. gr". Zie voetnoot 18 hierboven. Rekening van Clais Utenhove, baljuwkapitein van Biervliet over het jaar 1413. Voor de andere vermeldingen, zie bewijsstukken I en III. 


\section{BIJLAGEN}

\section{I}

1408. - Lijst van de vissers-stuurlui, die na daartoe toelating ontvangen te hebben, in plaats van te Vlissingen, hun lading kaakharing te Biervliet gelost hadden.

Uittreksel uit de rekening van Bouden Janssone, baljuw van Biervliet, over de periode 17 september 1408 - 24 april 1409, ingediend te Rijsel. Brussel, Algemeen Rijksarchief, Rekenkamer, nr 13662.

De Aernoud Weyte, maronnier qui avoit fait 3 las de harinc kake en la mer et venoit devant Flessinghen arriver en Zeelande, pour ce que le dit harinc est deffendus ou pays de Flandres par le poursieute daucuns merchans pissonniers de Flandres, le dit Arnoud envoya a Biervliet au bailli sil vaulsist accorder quil amenast le dit harinc devant le havene de Biervliet en payant de chascun tonnel $6 \mathrm{~s}$. par. pour monseigneur, ainsi quil soloient faire en temps passe a ma dame, qui dieux en ait lame, sans le dit harinc vendre ou estre despendus ou dit pays de Flandre. Et pour ce que le dit harinc fu vendus a Brabanchons et la despendus et ailleurs, se le accorde ledit bailli pour le pourfit de monseigneur et en recu du dit Arnoud de chascun tonnel 6 s., monte ala somme de $10 \mathrm{lb} .16 \mathrm{~s}$.

De Hughe le Costre, samlablement 12 tonnaulx, receu de chascun tonnel $6 \mathrm{~s}$, monte ala somme de $3 \mathrm{lb} .12 \mathrm{~s}$.

De Stoffel Wobracht, samlablement 6 tonnaulx, receu de chascun tonnel $6 \mathrm{~s}$., monte a la somme de $36 \mathrm{~s}$.

De Jehan Dankaerd, samlablement 18 tonnaulx de chascun tonnel $6 \mathrm{~s}$., monte a la somme de $5 \mathrm{lb} .8 \mathrm{~s}$.

II

1410. - Lijst van de vissers-stuurlui, die door de waterbaljuw van het $Z$ win beboet geworden waren, omdat ze hun lading kaakharing te Vlissingen gelost hadden.

Uittreksel uit de rekening van Pieter Gherbode, waterbaljuw van Mude, over de periode 22 september 1410 - 12 januari 1411. Rijsel, Archives départementales du Nord, Chambre des comptes de Flandre, nr 6117.

De Pieter Weite, pescheur de Cocxide, lequel fu calengie et pris par le dit bailli pour ce que a Vlissinghe en Zeelande il avoit mene et vendu noef tonneaulx de herens cacques appele contrefait herenc sans avoir paiie a Breiulet le noble de la last a mon- 
seigneur ou a son commis, dont selon les ordenances et estatus fait par mon dit seigneur il a fourfait $50 \mathrm{lb}$. parisis, comme plus aplain est fait mencion es lettres de mondit seigneur sur ce piecha faites, pour laquelle amende, ledit Pieter fu pris comme dit est et aussi pour le noble de mon dit seigneur. Aquoy le dit Pieter pria par son serment que de la costume ne savoit en ne cuidoit riens a ce meffaire et moult requist grace, le bailly a sa requeste, attendu que cest un pouvre pescheur cheuance de femme et denfans et sur mer de peschier comment quil gouverne lui, sa dite femme et enfans, a la pryere de Wouter de Grote, son hoste, atendu aussi que le (dist) Pieter promist a contenter le receveur de Flandre du noble de la lest, le receu en grace et composicion dicelle amende pour XXIIII lb.

De Jehan le Kien, pescheur de Blankenberghe, lequel pareillement fu pris par le dit bailli, pour ce quil avoit vendu au dit lieu de Vlissinghe troix lest et demi dudit herenc sans aussi selon lordenance de mon dit seigneur avoir paye audit lieu de Breiuliet un noble de chacune lest, dont pareillement 1 amende est $50 \mathrm{lb}$. parisis, de laquelle amende, atendu aussi cest un pouvre pescheur, ala priere de plusieurs bonnes gens et que ace il ne cuida riens maeffaire et aussi quil promist a contenter le receveur de Flandre, le bailli le receu en grace et composicion de la dite amende pour $25 \mathrm{lb}$.

\section{III}

1412. - Lijst van de stuurlui, die in 1412 te Biervliet Vlaamse kaakharing losten en het hiervoor verschuldigde "nobelgheld" stortten.

Uittreksel uit de rekening van Jehan Rugheroc, ontvanger van de tol te Biervliet, over de periode 12 september 1412 tot 21 juli 1413. Brussel, Algemeen Rijksarchief, Rekenkamer, $\operatorname{nr} 22522$, voorlaatste folio, verso.

Compte Jehan Rugheroc, commis par Godefroy Le Sauvage, receveur general de Flandres, a recevoir le droit, que monseigneur prent sur le herenc caque fait et caque sur la mer entre les jours St Bertelmi et St Maihieu par les pescheurs tant de Flandre comme autres estrainggiiers, dont mon dit seigneur prent de chacun lest ung noble selon les lettres dottroy de mon dit seigneur, que sur ce ont ceulx de la ville de Biervliet de tout ce quil a receu a la cause dite de lannee et saison 1412 et est le noble compte a $5 \mathrm{~s}$ gr. nouvelle monnaie de Flandre. 
(In de rand) Declaire quant ledit ottroy encommenca et quant il doit expirer.

Recepte :

De Jehan le Chien de Blanquenberghe pour 3 lest 2 barilz $15 \mathrm{~s}$. 10 d. gros.

De Guillaume Valke de Bieruliet pour 2 lest 4 barilz 11 s. 8 d. gr.

De Lem Duvel de Heys pour 17 barilz 7 s. 6 d. gr.

De Hughe le Costre de Bieruliet pour 3 lest 9 barilz et demi $18 \mathrm{~s}$. $9 \mathrm{~d}$. gr. et maille

De Maes filz Jehan de Bieruliet pour 6 barilz 2 s. 6 d. gr.

De Jehan le Chien de Blancqueberghe pour 9 barilz et demi 3 s. $11 \mathrm{~d}$. gr. et maille.

De Pieter le Gloeyer de Coxyde pour 3 lest 2 barilz 15 s. $10 \mathrm{~d}$. gr. De Christofre Wobrecht de Bieruliet pour 19 barilz 7 s. 9 d. groz.

De Jehan Danckaert de Hughevliete pour 4 lest 3 barilz 21 s. 3 d. $g r$.

Somme $5 \mathrm{lb} .5$ s. $6 \mathrm{~d}$. gros, valent en livre parisis fort $63 \mathrm{lb}$. $5 \mathrm{~s}$, (la quelle il doit rendre, sont rendu par le premier compte J. Utenhove en recepte commune et compte cy) (1).

IV

1412 maart 7, Parijs.

Ordonnantie van Jan zonder Vrees, hertog van Bourgondï, gericht aan de waterbaljuw te Sluis, evenals aan de baljuws te Brugge, Nieuwpoort, Oostende en Biervliet, waardoor de hertog aan de vissers van Vlaanderen, Zeeland en Holland toelating verleent onder bepaalde voor waarden, waaronder de betaling van één nobel per last, de kaakharing, die ze op zee gemaakt hebben, in voornoemde havens te lossen en te verkopen.

Kopie naar een cartularium. Rijsel, Archives départementales du nord, B 1282/15.407, papieren schrijfboek, folios 9 recto en verso en 10 recto.

Ottroy... (1) monseigneur de Bourgongne, conte de Flandre, aux maronniers pes(cheurs) de Flandre, quilz puissent vendre herenc caque... (1) es villes de Neufport, de Lescluse et dOstende, comme ilz ont acoustume de faire a Biervliet, en paiant ung noble de chacun lest.

Jehan, duc de Bourgogne, conte de Flandres etc. A noz bailliz

(1) Bijgevoegd.

(2) Onleesbaar wegens beschadiging. 
de Bruges, de leaue a Lescluse, de Neufport, dOestende, de Biervliet et a tous les autres baillis justiciers et officiers de notre dit conte et paiz de Flandres ou a leures lieuxtenans, salut. Receu avons lumble supplicacion des maronniers et pescheurs de nosdits pais et conte de Flandres, contenant que pour gouverner eulx, leures femmes et enfans et avoir leur vie et sustentacion, ils soient acoustumez de pescher par dela le lieu appelle le Wal et prendre chacun an du jour de la feste Saint Berthelemi jusques a la Saint Mahieu de herencs fres et aucune foiz les salez en tonneaulx et en faire harenc cacque bon et convenable et lequel, ainsi fait, ilz nont peu ne encore ne peuent, obstant certaine ordonnance japieca faite, vendre ne allouer en notre dit pais de Flandres sur peine dudit herenc estre fourfait a nous et sur lamende de 50 liv. par. de notre monnoye de Flandres toutes les foiz que eulx ou lun deulx seront trouvez faisans le contraire, fors seulement en notre ville de Biervliet, qui de ce ont obtenu grace et ottroy de nous par nos lettres patentes donnees en notre ville de Bruges le 7e jour de septembre lan M CCCC IX, comme par icelles plusaplain peut apparoir, pour illec amenez, vendre ou faire vendre parmi ce que de chacun lest desdits herencs, ilz paient ung noble a notre prouffit. Et il soit ainsi que souventeffoiz il advient que lesdits pescheurs ou les aucun deulx, qui en ladite saison ont prins du herenc frez, salent et caquent icellui sur la mer, les ungs plus les autres moins, et aucunement nen font en ung voiage que dix ou douze tonneaulz et arrivent chargiez de leur herenc fres, ensemble de cellui que ansi ilz ont sale et cacque, les aucuns a Neufport, les autres a Oostende et les autres au port delEscluse, ou desdits lieux il leur convient envoier ledit herenc caque a grant dangiez et despense en notre dite ville de Biervliet et mainteffoiz pour ce dangiez delaissent a caquer de leur dit frez herenc, parquoy le noble, que nous avons sur le lest dicellui herenc vendu audit Biervliet, ne nous revient pas a se grant valeurs, quil seroit en esdits lieux de Neufport, de Lescluse et dOstende, ilz (silz ?) pooient franchement et sauvement sanz meffaire mener, vendre et allouer leur dit herenc caque aussi bien que ilz font audit lieu de Biervliet, si comme ilz dient en nous suppliant notre grace et permission sur ce. Pourquoy nous desirans lavancement du... (1) et mesmement desdits pescheurs, nos subgiez, qui sont moult povres gens, inclinans favorablement a leurdit supplicacion, deliberacion sur ce eue avec plusieurs des gens de notre conseil, avons de grace especial, les choses dessusdit conside-

(1) Onleesbaar. 
rees, octtroye et voulu, ottroyons et nous plaist que, non obstant lesdits ordonnance et deffense par nous faite, lesdits maronniers et pescheurs de notredit pais de Flandres et chacun deulx, de quelque lieu quilz soient, puissent decyevant jusques a notre rappel alez pescher et prendre du herenc fres par dela ledit lieu appelle le Wal tous les ans depuis ledit jour Saint Berthelemi jusques audit jour Saint Mahieu et icellui herenc frez faire et salez, mettre en tonneaulx et en faire herenc caque, le amenez esdits lieux delEscluse, Neufport, dOostende et de Biervliet et illec et non ailleurs en notredit pais de Flandres le vendre a ceulx, qui achetez le vouldront, sur peine dudit herenc par eulx prins et caque, comme dit est, estre fourfait et acquis envers nous et sur lamende de cinquante livres parisis de notre dit monnaie de Flandres, toutes les foiz que eulx ou lun deulx soient trouvez forfais ou avoir fait le contraire. Et moiennant ce ilz sont tenuz de paier pour nous a notre proffit aux commis a ce de par nous en chacune desdits lieux cy dessus nommez ou ilz arriveront, deschargeront et venderont leurdit herenc caque pour chacun lest ung noble dor, ainsi que len a fait audit lieu de Biervliet, pourveu que chacun pescheur, arrivant et deschargant tel herenc es lieux dessusdit ou en lun diceulx, sera tenu sur les mesmes peines et amendes de signiffier ou faire savoir par ung de ses gens a cellui ou a ceulx, qui devers nous seront commis a recevoir notre dit droit, au juste quele quantite dudit herenc cacque ilz auroit en leurs nefs et avant que icellui ilz puissent deschargiez, vendre ne allouer. Et avec ce seront tenuz de mettre aux tonneaulx, esquelz ilz le auront sale, enseigne publique tele que les bailli et loy desdits lieux fera ordonnez, afin que aucun nen soit fraude ne deceu. Et avec ce ceulx, qui les acheteront, feront caucion sur semblables peines et amendes, de les non mener, vendre ne faire vendre, ne despenser autrepart dedens nosdits pais et conte. Et se il avenoit que par fortune, oraige et tempeste de temps ou de vent contraire il convenait que lesdits pescheurs ou les aucuns deulx alassent arriver ou royaume dAngleterre, nous leur ottroyons et nous plaist, que lesdits herens, ainsi salez et mis en tonneaulx, ilz puissent vendre illec sanz pour ce chence meffaire envers nous. Sauf que apres la vendicion diceulx herens eulx, retournez en leur desusdits quatre places, les (maistres) (1) et compaignons feront illec serment, par lequel ilz seront creuz de la venue dudit orrage et tempeste de temps et du nombre, quantite dudit herenc, qui par eulx y aura este vendu, duquel ils nous se-

(1) Onleesbaar wegens beschadiging. 
ront tenuz de paier ung noble dor de chacun lest. Et si par semblable cas et fortune leur fausist arriver en Meuse en Westerscoude ou autrepart en paiz de Zeellande, si seront ilz tenuz sur la meisme peine de amenez ou faire amenez ledit herenc caque en lun desdits quatre lieux, assavoir est Lescluse, Neufport, Oostende et Biervliet. Reserve que se ilz avoient ou amenoient esdits lieux de Zeellande aucuns herens frez et non salez, ilz les y pourront vendre sanz meffaire. Enoultre, si aucuns pescheurs des paiz de Hollande de Zeellande ou d'autres lieux et pais vueillent venir et amener vendre tel herenc caque en aucun desdits lieux en nosdits pais et conte de Flandre par la maniere que dit est, faire le pourront en paiant de et pour chacun lest ung noble a notre prouffit, comme feront ceulx de nosdits pais et conte de Flandres. Se vous mandons et communiquons estroitement et a chacun de vous, si comme a lui appartendra, que de nosdit grace et ottroy jusques a notre rappel et par la maniere dessus declaire, vous faites et souffrer et laissez lesdits maronniers et pescheurs de notredit pais de Flandres et pareillement ceulx desdits pais de Hollande et Zeellande et dautres lieux et chacun deulx, selon ce que dessus est touchie, plainement et paisiblement joir et user sanz leur faire donner, ne mettre, ne souffrir estre fait, donne ou mis, ne a aucun deulx aucun empeschement ou destourbier au grace en executant chacun de vous endroit soy pour et a notre prouffit les dits peines et amendes par toute voyes et maniere deues et raisonnables de et sur tous ceulx qui transgresseront ou yront outre la tennuer de ces presentes nos lettres sairements et grace, sanz deport ou difficulte aucuns et notredit ottroy publie ou feras publiez es lieus de vos officiers par tous les lieux quil appartiendra se besoing est et requis en estes. Donne a Paris le VIIe jour de mars lan de grace M CCCC et onze.

Ainsi signe par monseigneur le duc a relacion... (1) de Saulx.

Cest copie a este extraite du registre des chartres estant en la chambre des comtes a Lille, commencant en lan M CCCC XII, folio XXX.

(1) Onleesbare aftrorting. 